\title{
Anisotropy of HARDI Diffusion Profiles Based on the $\mathrm{L}^{2}$-Norm
}

\author{
Philipp Landgraf ${ }^{1}$, Dorit Merhof ${ }^{1}$, Mirco Richter ${ }^{1}$ \\ ${ }^{1}$ Institute of Computer Science, Visual Computing Group, University of Konstanz \\ philipp.landgraf@uni-konstanz.de
}

\begin{abstract}
The fractional anisotropy (FA) value for Diffusion Tensor Imaging is widely used to determine the anisotropy of diffusion in a given voxel. As the FA value is based on the tensor's eigenvectors it is not possible to calculate this quantity for HARDI diffusion profiles. In this paper we introduce an anisotropy index for HARDI data that utilizes the $\mathrm{L}^{2}$-norm as the most natural notion of distance for square-integrable functions on the two-sphere such as HARDI diffusion profiles and show that it is the limit of the generalized fractional anisotropy (GFA) index. Our index is well-defined and rotationally invariant and thus resolves the unsatisfactory issues with the GFA index.
\end{abstract}

\section{Introduction}

The gray matter in the human brain comprises about 10 billion neuronal cells that use their axons to transmit information. These axons form bundles called neuronal fiber tracts that connect functional areas.

The localization of white matter tracts is of great interest for neurological research about brain structure and function, as well as for neurosurgery to preserve important tract systems during surgical intervention.

The noninvasive and in vivo reconstruction of fiber tracts is possible with Diffusion MRI. This is a medical imaging modality that is sensitive to the random thermal movement of water molecules. This information allows for inference about the structure of the tissue in the human brain because diffusion tends to be hampered in directions orthogonal to fiber bundles.

A widely used model to describe the measured diffusion is the diffusion tensor [1] which is the covariance matrix of a three-dimensional Gaussian distribution and is used to model the diffusion orientation distribution function (ODF).

The fractional anisotropy value (FA) is a scalar measure derived from the diffusion tensor and quantifies the anisotropy of diffusion. As diffusion tends to be more anisotropic in white matter this value can be applied to distinguish white matter from gray matter within the brain. Tracking algorithms therefore often operate with an FA threshold to prevent fiber tracking algorithms from leaving regions of white matter as in [2].

However, the diffusion tensor can only resolve a single diffusion direction per voxel. To overcome this deficit high angular resolution diffusion imaging 
(HARDI) techniques such as Q-Ball imaging [3] or higher order tensors [4] have been developed that increase the number of gradients and reconstruct an apparent diffusion coefficient (ADC) profile at each voxel.

The FA value is based on the eigenvalues of the diffusion tensor. Therefore, it is not possible to calculate the FA value of a general ADC profile due to its lack of eigenvalues. Several anisotropy indices for HARDI diffusion profiles can be found in the literature, such as the indices of Frank [5] and Chen [6].

In this paper we generalize the popular GFA index [3] that for instance was employed to investigate the genetic effects on brain fiber connectivity [7]. Nevertheless it has some theoretical limitations which are resolved in this work.

\section{Materials and Methods}

This section starts with the definition of the GFA index and its drawbacks. Thereafter the L-index is defined.

\subsection{The GFA index}

The GFA index defined by Tuch [3] is a straightforward extension of the FA value to HARDI ODFs. After picking directions $\left\{x_{i}\right\}_{i=1, \ldots, n}$ one can define

$$
\operatorname{GFA}_{n}(f)=\sqrt{\frac{n \sum_{i=1}^{n}\left(f\left(x_{i}\right)-\langle f\rangle_{n}\right)^{2}}{(n-1) \sum_{i=1}^{n} f\left(x_{i}\right)^{2}}}
$$

where $\langle f\rangle_{n}=\frac{1}{n} \sum_{i=1}^{n} f\left(x_{i}\right)$ is the mean value.

This index is automatically scaled to the unit interval and maps isotropic ODFs to zero. In the special case of diffusion tensors, GFA reduces to the FA value if the chosen directions happen to be the eigenvectors of the tensor.

The GFA index has, however, some severe drawbacks. First of all, it is not well-defined as it strongly depends on the number and choice of the directions in which the function is evaluated. Secondly, GFA $(f)$ is not rotationally invariant as one would expect from an anisotropy index.

\subsection{The L-Index}

The basic idea of our anisotropy index is to measure how spherical the ADCprofile is. The ODF is expressed as a linear combination of spherical harmonics that are an orthonormal basis of the Hilbert space $\mathrm{L}^{2}$ of square-integrable functions. The natural measure of distance in this space is the $\mathrm{L}^{2}$-norm $\|\cdot\|_{2}$.

If the mean value $\langle f\rangle$ of $f$ defined by

$$
\langle f\rangle=\frac{1}{2 \pi^{2}} \int_{0}^{\pi} \int_{0}^{2 \pi} f(\theta, \varphi) d \varphi d \theta
$$


and the corresponding constant function (which we also denote by $\langle f\rangle$ ) is taken as the closest approximation of $f$ by a sphere, the $\mathrm{L}^{2}$ distance $\|f-\langle f\rangle\|_{2}$ between $f$ and $\langle f\rangle$ contains the information about how close $f$ resembles a sphere. Normalizing with the $\mathrm{L}^{2}$ norm of $f$ yields the L-index

$$
L(f)=\frac{\|f-\langle f\rangle\|_{2}}{\|f\|_{2}}
$$

\section{Results}

The main result of this paper is that the L-index is the limit of the $\mathrm{GFA}_{n}$ index as $n$ increases if one chooses the $x_{i}$ as rectilinear grid points in the $[0, \pi) \times[0,2 \pi)$ domain.

Theorem 1. $\lim _{n \rightarrow \infty} G F A_{n}(f)=L(f)$

Proof. Let $f:[0, a] \times[0, b] \rightarrow \mathbb{R}$ be a real valued function on a rectangle. Define $\operatorname{grid}$ points $x_{i j}^{(n)}:=\left(i \frac{a}{n}, j \frac{b}{n}\right), i, j=0, \ldots, n$ for each $n \in \mathbb{N}$ Furthermore let

$$
\Phi(f, n)=\sum_{i=1}^{n} \sum_{j=1}^{n} \frac{a b}{n^{2}} f\left(x_{i j}^{(n)}\right)^{2}
$$

Now consider the characteristic functions $\chi_{A_{i j}^{(n)}}:[0, a] \times[0, b] \rightarrow\{0,1\}$ for the sets $A_{i j}^{(n)}=\left[(i-1) \frac{a}{n}, i \frac{a}{n}\right) \times\left[(j-1) \frac{b}{n}, j \frac{b}{n}\right)$. The characteristic function $\chi_{A}$ of a set $A$ is defined by $\chi_{A}(x)=1$ if $x \in A$ and $\chi_{A}(x)=0$ otherwise. Then we have

$$
\Phi(f, n)=\int_{0}^{a} \int_{0}^{b} \sum_{i=1}^{n} \sum_{j=1}^{n} f\left(x_{i j}^{(n)}\right)^{2} \chi_{A_{i j}^{(n)}}(x, y) d y d x
$$

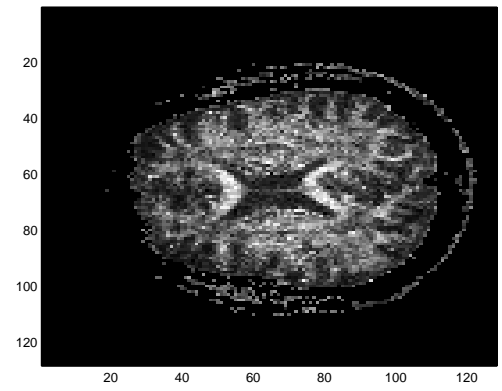

(a) FA value

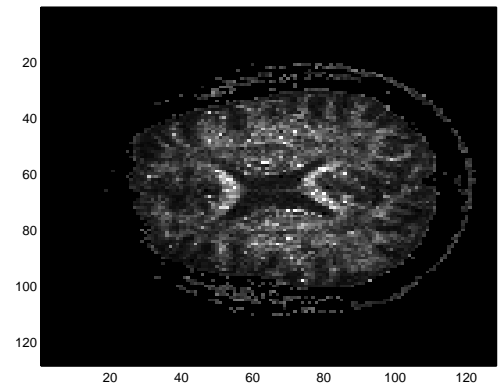

(b) L-index

Fig. 1. (a) FA values of a single slice from the dataset, (b) L-index values from the same slice capped at 0.5 for enhancement of contrast. 
which converges to $\|f\|_{2}^{2}=\int_{0}^{a} \int_{0}^{b} f(x, y)^{2} d y d x$ as the sum describes a step function that converges to $f^{2}$.

A similar argument shows that $\lim _{n \rightarrow \infty}\langle f\rangle_{n}=\langle f\rangle$. Together this yields the convergence because

$$
\lim _{n \rightarrow \infty} \operatorname{GFA}_{n}(f)=\lim _{n \rightarrow \infty} \sqrt{\frac{n}{n-1}} \frac{\sqrt{\Phi\left(f-\langle f\rangle_{n}, n\right)}}{\sqrt{\Phi(f, n)}}=\frac{\|f-\langle f\rangle\|_{2}}{\|f\|_{2}}
$$

So basically $\operatorname{GFA}_{n}(f)$ is an approximation to the true anisotropy value $L(f)$ using the rectangle rule for numerical integration. Note that $\operatorname{GFA}_{n}(f) \in[0,1]$ implies $L(f) \in[0,1]$ as well. Furthermore, this index is invariant under scaling with a scalar, i.e. $L(c f)=L(f)$ as one would expect from a measure of shape. Additionally, it is well defined and rotationally invariant since it is defined by integration over the sphere and thus resolves the drawbacks of the GFA index.

\subsection{Application to human brain data}

The Diffusion MRI dataset used in this work was provided for the IEEE Visualisation Contest 2010. It is courtesy of Prof. B. Terwey, Klinikum Mitte, Bremen, Germany and was acquired on a Siemens 3T Verio MR scanner. 30 gradient directions and two averages per gradient were acquired with $b=1000 \mathrm{~s} / \mathrm{mm}^{2}$.

ADC-profiles were reconstructed with maximal order $M=6$ as in [8] with regularization parameter $\lambda=0.5$. Subsequently, diffusion tensors were reconstructed by a least squares fit to calculate FA values.

Figure 1 shows the FA values (a) and L-index values (b) of a single slice in the dataset. Note that due to the fact that the distribution of the L-index is relatively narrow the values have been thresholded at 0.5 for contrast enhancement. From the histograms over the whole dataset for FA values and the L-index, we conclude that 0.5 is a reasonable threshold (Fig. 2).

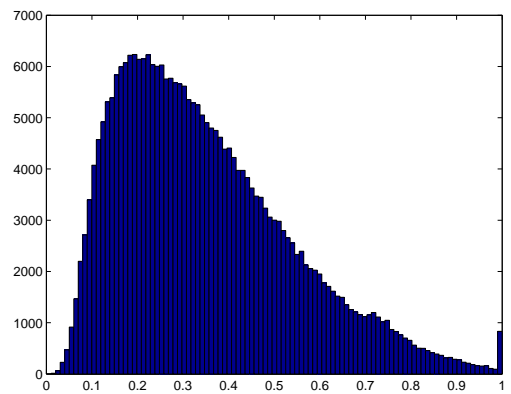

(a) FA value

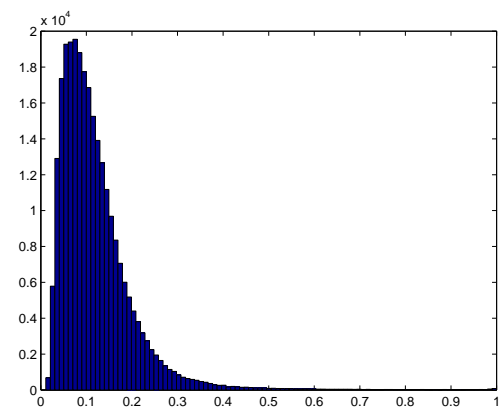

(b) L-index

Fig. 2. Histogram of the FA values (left) and L-index (right) over the entire dataset. For the sake of clarity, zero voxels were left out. 
The correlation coefficient $\operatorname{corr}(\mathrm{L}-\mathrm{index}, \mathrm{FA})=0.9576$ shows a strong (positive) linear correlation between the L-index and the FA value.

\section{Discussion}

We showed that the L-index resolves the unsatisfactory issues with the GFA index not being well-defined nor rotationally invariant. Moreover the GFA index can be interpreted as approximation of the L-index by numerical integration.

Due to the strong linear correlation between the FA value and the L-index the latter can be used to segment white matter in the brain or serve as a stopping criterion for tracking algorithms based on HARDI data.

\section{References}

1. Basser PJ, Mattiello J, LeBihan D. MR diffusion tensor spectroscopy and imaging. Biophys J. 1994;66(1):259-67.

2. Merhof D, Sonntag M, Enders F, et al. Visualization of diffusion tensor data using evenly spaced streamlines. In: Proc VMV; 2005. p. 257-64.

3. Tuch DS. Q-ball imaging. Magn Reson Med. 2004;52(6):1358-72.

4. Özarslan E, Mareci TH. Generalized diffusion tensor imaging and analytical relationships between diffusion tensor imaging and high angular resolution diffusion imaging. Magn Reson Med. 2003;50(5):955-65.

5. Frank LR. Characterization of anisotropy in high angular resolution diffusionweighted MRI. Magn Reson Med. 2002;47(6):1083-99.

6. Chen Y, Guo W, Zeng Q, et al. Estimation, smoothing, and characterization of apparent diffusion coefficient profiles from high angular resolution DWI. Proc IEEE CVPR. 2004;1.

7. Chiang MC, Barysheva M, Lee AD, et al. Mapping genetic influences on brain fiber architecture with high angular resolution diffusion imaging (HARDI). Proc IEEE ISBI. 2008;5:871-4.

8. Descoteaux M, Angelino E, Fitzgibbons S, et al. Apparent diffusion coefficients from high angular resolution diffusion imaging: estimation and applications. Magn Reson Med. 2006;56(2):395-410. 